PROCEEDINGS OF THE

AMERICAN MATHEMATICAL SOCIETY

Volume 134, Number 6, Pages 1687-1699

S 0002-9939(05)08158-X

Article electronically published on December 2, 2005

\title{
A LOCAL LIFTING THEOREM FOR SUBNORMAL OPERATORS
}

\author{
WITOLD MAJDAK, ZOLTÁN SEBESTYÉN, JAN STOCHEL, AND JAMES E. THOMSON
}

(Communicated by Joseph A. Ball)

\begin{abstract}
Criteria for the existence of lifts of operators intertwining subnormal operators are established. The main result of the paper reduces lifting questions for general subnormal operators to questions about lifts of cyclic subnormal operators. It is shown that in general the existence of local lifts (i.e. those coming from cyclic parts) for a pair of subnormal operators does not imply the existence of a global lift. However this is the case when minimal normal extensions of subnormal operators in question are star-cyclic.
\end{abstract}

\section{INTRODUCTION}

The first characterization of operators which lift to the commutant of a minimal normal extension of a subnormal operator was formulated by Bram [3, Theorem 7] (Yoshino observed in [23] that the commutativity assumption in Bram's theorem is superfluous). Afterwards it was extended to the case of intertwining operators by Embry [8] (see also [1, 5, 21] and the references therein). The present paper provides new characterizations of liftable operators. Our solutions are based on Mlak's version of a result of Maserick [13. Two of them, namely parts (iii $\mathcal{M}_{\mathcal{M}}$ ) and $\left(\right.$ iv $_{\mathcal{M}}$ ) of Theorem [3.2, deal with the multicyclic case. They are no longer true without assuming $T S_{1}=S_{2} T$. Theorem 3.5 improves Embry's solution of the lifting commutant problem [8. Again the assumption $T S_{1}=S_{2} T$ turns out to be indispensable. In Theorem 4.2 we prove that an intertwining operator between subnormal operators lifts to an intertwining operator between their minimal normal extensions if and only if (1) the restriction of the intertwining operator to each cyclic invariant subspace lifts, and (2) the supremum of the norms of the cyclic lifts is finite. Example 5.3 shows that condition (1) is not by itself sufficient. In the case of star-cyclic minimal normal extensions the hypothesis (2) can be dropped; cf. Theorem 6.2. The inspiration for writing this paper comes from [19].

\section{Preliminaries}

In what follows, $\mathbb{N}$ stands for the additive semigroup of all nonnegative integers. All linear spaces taken into consideration in this paper are assumed to be complex.

Received by the editors July 8, 2004 and, in revised form, January 10, 2005.

2000 Mathematics Subject Classification. Primary 47B20; Secondary 47A20.

Key words and phrases. Subnormal operator, minimal normal extension, star-cyclic minimal normal extension, lift of intertwining operator, lifting commutant theorem.

The research of the third author was supported by KBN grant 2 P03A 037024. 
Given Hilbert spaces $\mathcal{H}$ and $\mathcal{K}$, we denote by $\boldsymbol{B}(\mathcal{H}, \mathcal{K})$ the set of all bounded linear operators from $\mathcal{H}$ into $\mathcal{K}$. For simplicity we write $\boldsymbol{B}(\mathcal{H})$ instead of $\boldsymbol{B}(\mathcal{H}, \mathcal{H}) ; I_{\mathcal{H}}$ is the identity operator on $\mathcal{H}$. If $\mathcal{H} \subseteq \mathcal{K}, S \in \boldsymbol{B}(\mathcal{H}), T \in \boldsymbol{B}(\mathcal{K})$ and $S f=T f$ for all $f \in \mathcal{H}$, then we write $S \subseteq T$. We denote by $\operatorname{lin} X$ (resp. $\bigvee X$ ) the linear span (resp. the closed linear span) of a subset $X$ of $\mathcal{H}$. An operator $S \in \boldsymbol{B}(\mathcal{H})$ is said to be subnormal if there exists a Hilbert space $\mathcal{K}$ and a normal operator $N \in \boldsymbol{B}(\mathcal{K})$ such that $S \subseteq N$; such $N$ is called a normal extension of $S$. If additionally $\mathcal{K}=$ $\bigvee\left\{N^{* n} f: f \in \mathcal{H}, n \in \mathbb{N}\right\}$, then $N$ is called a minimal normal extension of $S$ (see [5] for more details).

A commutative semigroup $(\Omega,+)$ is said to be a $*$-semigroup if it is equipped with a mapping $*: \Omega \rightarrow \Omega$, called an involution on $\Omega$, such that $(\xi+v)^{*}=\xi^{*}+v^{*}$ and $\left(\xi^{*}\right)^{*}=\xi$ for all $\xi, v \in \Omega$. An operator-valued function $\omega: \Omega \rightarrow \boldsymbol{B}(\mathcal{H})$ defined on a commutative $*$-semigroup $(\Omega,+, *)$ is said to be positive definite if

$$
\sum_{m, n=0}^{k}\left\langle\omega\left(v_{n}^{*}+v_{m}\right) f_{m}, f_{n}\right\rangle \geqslant 0, \quad\left\{v_{m}\right\}_{m=0}^{k} \subseteq \Omega,\left\{f_{m}\right\}_{m=0}^{k} \subseteq \mathcal{H}, k \in \mathbb{N} .
$$

We say that $\omega$ is weakly positive definite if for every vector $f$ in $\mathcal{H}$, the scalar function $\langle\omega(\cdot) f, f\rangle$ is positive definite on $\Omega$.

For further considerations it will be convenient to state beforehand, for easy reference, the following variant of the Maserick theorem (cf. [13, Theorem 3.2]) which is due to Mlak (cf. [14, Proposition 9.6]; see also [18, Corollary 4] and 20, Theorem 6.1] for generalizations).

Theorem 2.1. Let $(\Omega,+, *)$ be a commutative $*$-semigroup with zero and let $\omega$ : $\Omega \rightarrow \boldsymbol{B}(\mathcal{H})$ be an operator-valued function such that $\sup _{v \in \Omega}\|\omega(v)\|<\infty$. Then $\omega$ is positive definite if and only if it is weakly positive definite.

\section{CRiteria FOR the EXISTENCE OF Lifts}

Suppose that:

$\mathcal{H}_{j}$ is a closed linear subspace of a Hilbert space $\mathcal{K}_{j}$ and $S_{j} \in \boldsymbol{B}\left(\mathcal{H}_{j}\right)$ is a subnormal operator with a minimal normal extension $N_{j} \in \boldsymbol{B}\left(\mathcal{K}_{j}\right), j=1,2$.

Definition 3.1. Put $\mathcal{I}\left(N_{1}, N_{2}\right)=\left\{R \in \boldsymbol{B}\left(\mathcal{K}_{1}, \mathcal{K}_{2}\right): R N_{1}=N_{2} R\right\}$. We say that an operator $T \in \boldsymbol{B}\left(\mathcal{H}_{1}, \mathcal{H}_{2}\right)$ lifts to $\mathcal{I}\left(N_{1}, N_{2}\right)$ if there exists an operator $\widehat{T} \in \mathcal{I}\left(N_{1}, N_{2}\right)$ such that $T \subseteq \widehat{T} ; \widehat{T}$ is called a lift of $T$.

Note that such a $\widehat{T}$ is unique. Indeed, by the Putnam-Fuglede theorem [15], $\widehat{T} N_{1}^{*}=N_{2}^{*} \widehat{T}$ and consequently

$$
\widehat{T} N_{1}^{* n} f=N_{2}^{* n} \widehat{T} f=N_{2}^{* n} T f, \quad f \in \mathcal{H}_{1}, n \in \mathbb{N},
$$

which by the minimality of $N_{1}$ determines $\widehat{T}$ uniquely (see also [3, Theorem 7]).

The following theorem plays a significant role in further investigations.

Theorem 3.2. Let $\mathcal{H}_{j}, \mathcal{K}_{j}, S_{j}$ and $N_{j}, j=1,2$, be as in (3.1). Suppose that $\mathcal{M}$ is a linear subspace of $\mathcal{H}_{1}$ such that $\mathcal{H}_{1}=\bigvee\left\{S_{1}^{n} f: f \in \mathcal{M}, n \in \mathbb{N}\right\}$. Let $T \in \boldsymbol{B}\left(\mathcal{H}_{1}, \mathcal{H}_{2}\right)$. Then the following conditions are equivalent:

(i) $T$ lifts to $\mathcal{I}\left(N_{1}, N_{2}\right)$, 
(ii) there exists a real number $c \geqslant 0$ such that

$$
\sum_{m, n=0}^{k}\left\langle S_{2}^{m} T f_{n}, S_{2}^{n} T f_{m}\right\rangle \leqslant c \sum_{m, n=0}^{k}\left\langle S_{1}^{m} f_{n}, S_{1}^{n} f_{m}\right\rangle
$$

for all finite sequences $\left\{f_{m}\right\}_{m=0}^{k} \subseteq \mathcal{H}_{1}$,

(iii) there exists a real number $c \geqslant 0$ such that

$$
\sum_{m, n, p, q=0}^{k}\left\langle S_{2}^{n+p} T f_{m, n}, S_{2}^{m+q} T f_{p, q}\right\rangle \leqslant c \sum_{m, n, p, q=0}^{k}\left\langle S_{1}^{n+p} f_{m, n}, S_{1}^{m+q} f_{p, q}\right\rangle
$$

for all finite 2-sequences $\left\{f_{m, n}\right\}_{m, n=0}^{k} \subseteq \mathcal{H}_{1}$,

(iii $\mathcal{M}_{\mathcal{M})} T S_{1}=S_{2} T$ and there exists a real number $c \geqslant 0$ such that (3.3) holds for all finite 2-sequences $\left\{f_{m, n}\right\}_{m, n=0}^{k} \subseteq \mathcal{M}$,

(iv) there exists a real number $c \geqslant 0$ such that

$$
\sum_{m, n, p, q=0}^{k}\left\langle S_{2}^{n+p} T f, S_{2}^{m+q} T f\right\rangle \lambda_{m, n} \overline{\lambda_{p, q}} \leqslant c \sum_{m, n, p, q=0}^{k}\left\langle S_{1}^{n+p} f, S_{1}^{m+q} f\right\rangle \lambda_{m, n} \overline{\lambda_{p, q}}
$$

for every $f \in \mathcal{H}_{1}$ and for all finite 2 -sequences $\left\{\lambda_{m, n}\right\}_{m, n=0}^{k} \subseteq \mathbb{C}$,

(iv ${ }_{\mathcal{M})} T S_{1}=S_{2} T$ and there exists a real number $c \geqslant 0$ such that (3.4) holds for every $f \in \mathcal{M}$ and for all finite 2-sequences $\left\{\lambda_{m, n}\right\}_{m, n=0}^{k} \subseteq \mathbb{C}$.

Moreover, if (i) holds, then

(v) $T S_{1}=S_{2} T$,

(vi) the smallest real number $c \geqslant 0$ satisfying condition (ii) (resp. (iii), (iii $\mathcal{M}_{\mathcal{M}}$ ), (iv), (iv $\mathcal{M})$ ) is equal to $\|\widehat{T}\|^{2}$.

Remark 3.3. The question arises as to whether Theorem 3.2 still remains valid if we replace condition (ii) by its weak version:

$\left(\mathrm{ii}_{\mathrm{w}}\right)$ there exists a real number $c \geqslant 0$ such that

$$
\sum_{m, n=0}^{k}\left\langle S_{2}^{m} T f, S_{2}^{n} T f\right\rangle \lambda_{n} \overline{\lambda_{m}} \leqslant c \sum_{m, n=0}^{k}\left\langle S_{1}^{m} f, S_{1}^{n} f\right\rangle \lambda_{n} \overline{\lambda_{m}}
$$

for every $f \in \mathcal{H}_{1}$ and for all finite sequences $\left\{\lambda_{m}\right\}_{m=0}^{k} \subseteq \mathbb{C}$.

The answer is negative. Indeed, take any subnormal operator $S \in \boldsymbol{B}(\mathcal{H})$ for which there exists an operator $T \in \boldsymbol{B}(\mathcal{H})$ in the commutant of $S$ which does not lift to the commutant of a minimal normal extension of $S$ (cf. [5, Chapter II, §10]). Set $S_{1}=S_{2}:=S$. Then ( $\mathrm{ii}_{\mathrm{w}}$ ) is valid for $c=\|T\|^{2}$ because

$$
\begin{aligned}
\sum_{m, n=0}^{k}\left\langle S_{2}^{m} T f, S_{2}^{n} T f\right\rangle \lambda_{n} \overline{\lambda_{m}} & =\left\|T\left(\sum_{m=0}^{k} \overline{\lambda_{m}} S_{1}^{m} f\right)\right\|^{2} \\
& \leqslant\|T\|^{2}\left\|\sum_{m=0}^{k} \overline{\lambda_{m}} S_{1}^{m} f\right\|^{2}=\|T\|^{2} \sum_{m, n=0}^{k}\left\langle S_{1}^{m} f, S_{1}^{n} f\right\rangle \lambda_{n} \overline{\lambda_{m}} .
\end{aligned}
$$

Remark 3.4. Regarding Theorem 3.2, note that the implication (iii $\left.\mathcal{M}_{\mathcal{M}}\right) \Rightarrow(\mathrm{i})$ is no longer true if the hypothesis $T S_{1}=S_{2} T$ is dropped (though the equivalence $\left(\right.$ iii $\left._{\mathcal{M}}\right) \Leftrightarrow\left(\right.$ iv $\left._{\mathcal{M}}\right)$ still holds; see the proof of Theorem 3.2). To see this consider the isometric unilateral shift $S \in \boldsymbol{B}(\mathcal{H})$ given by $S e_{j}=e_{j+1}$ for $j \geqslant 0$, where $\left\{e_{j}\right\}_{j=0}^{\infty}$ 
is an orthonormal basis of a Hilbert space $\mathcal{H}$. Then $\mathcal{H}=\bigvee\left\{S^{n} f: f \in \mathcal{M}, n \in \mathbb{N}\right\}$ for $\mathcal{M}:=\mathbb{C} e_{0}$. Set $S_{1}=S_{2}:=S$ and $T:=S^{*}$. Since $T e_{0}=0$, we conclude that the inequality (3.3) holds for all finite 2-sequences $\left\{f_{m, n}\right\}_{m, n=0}^{k} \subseteq \mathcal{M}$ (with arbitrary $c \geqslant 0$ ), whereas condition (i) is not fulfilled (because $T S \neq S T$ ).

Proof of Theorem 3.2. First, note that without any loss of generality we can assume that the operators $S_{1}$ and $S_{2}$ are contractions. Indeed, if they are not, it suffices to replace $S_{1}, S_{2}, N_{1}$ and $N_{2}$ by $\frac{1}{a} S_{1}, \frac{1}{a} S_{2}, \frac{1}{a} N_{1}$ and $\frac{1}{a} N_{2}$, respectively, where $a$ is a positive real number such that $a \geqslant \max \left\{\left\|S_{1}\right\|,\left\|S_{2}\right\|\right\}$.

If (i) holds, then (v) is valid because

$$
T S_{1} f=\widehat{T} S_{1} f=\widehat{T} N_{1} f=N_{2} \widehat{T} f=N_{2} T f=S_{2} T f, \quad f \in \mathcal{H}_{1} .
$$

(i) $\Leftrightarrow($ ii) This is a substantial part of Theorem 10 of $[8$.

(ii) $\Rightarrow$ (iii) In view of (ii) $\Rightarrow$ (i) and (i) $\Rightarrow(v)$, we have $T S_{1}=S_{2} T$. Substituting $f_{m}=\sum_{q=0}^{k} S_{1}^{q} f_{m, q}$ into (3.2) and employing $T S_{1}=S_{2} T$, we get (3.3) after making the appropriate change of the summation indices.

(iii) $\Rightarrow$ (ii) Applying (3.3) to $f_{m, n}=\delta_{n, 0} f_{m}$ we obtain (3.2).

We now show that conditions $\left(\right.$ iii $_{\mathcal{M}}$ ) and (iv $\mathcal{M}_{\mathcal{M}}$ ) are equivalent without assuming that $T S_{1}=S_{2} T$. Indeed, substituting $f_{m, n}=\lambda_{m, n} f$ into (3.3), we get (3.4). For the converse, denote by $\Omega$ the $*$-semigroup $(\mathbb{N} \times \mathbb{N},+, *)$ with the coordinatewise defined addition and with the involution $(m, n)^{*}=(n, m), m, n \in \mathbb{N}$. There is no loss of generality in assuming that $\mathcal{M}$ is a closed linear subspace of $\mathcal{H}_{1}$. Since (3.4) is supposed to hold for every $f \in \mathcal{M}$ and for all finite 2-sequences $\left\{\lambda_{m, n}\right\}_{m, n=0}^{k} \subseteq \mathbb{C}$, the operator-valued function $\omega_{c}: \Omega \longrightarrow \boldsymbol{B}(\mathcal{M})$ defined by

$$
\omega_{c}(m, n)=\left.P\left(c S_{1}^{* m} S_{1}^{n}-T^{*} S_{2}^{* m} S_{2}^{n} T\right)\right|_{\mathcal{M}}, \quad m, n \in \mathbb{N},
$$

where $P$ is the orthogonal projection of $\mathcal{H}_{1}$ onto $\mathcal{M}$, is weakly positive definite and uniformly bounded (as $\left\|S_{1}\right\|,\left\|S_{2}\right\| \leqslant 1$ ). By virtue of Theorem $2.1 \omega_{c}$ is positive definite, which means that (3.3) holds for all finite 2-sequences $\left\{f_{m, n}\right\}_{m, n=0}^{k} \subseteq \mathcal{M}$.

The above applied to $\mathcal{M}=\mathcal{H}_{1}$ yields the equivalence (iii) $\Leftrightarrow$ (iv).

$\left(\right.$ iii $\left._{\mathcal{M}}\right) \Rightarrow$ (ii) Take a finite sequence $\left\{f_{m}\right\}_{m=0}^{k} \subseteq \operatorname{lin}\left\{S_{1}^{n} f: f \in \mathcal{M}, n \in \mathbb{N}\right\}$. Then there exist finite sequences $\left\{f_{m, q}\right\}_{q=0}^{l} \subseteq \mathcal{M}, m=0, \ldots, k$, such that

$$
f_{m}=\sum_{q=0}^{l} S_{1}^{q} f_{m, q}, \quad m=0, \ldots, k .
$$

Without loss of generality we can assume that $l=k$. Applying $T S_{1}=S_{2} T$, one can infer (3.2) from (3.3). Since the linear space $\operatorname{lin}\left\{S_{1}^{n} f: f \in \mathcal{M}, n \in \mathbb{N}\right\}$ is dense in $\mathcal{H}_{1}$, one can extend the inequality (3.2) over all finite sequences $\left\{f_{m}\right\}_{m=0}^{k}$ with entries in $\mathcal{H}_{1}$. This justifies the validity of $\left(\operatorname{iii}_{\mathcal{M}}\right) \Rightarrow($ ii).

Since (ii) implies both (iii) and $T S_{1}=S_{2} T$, we conclude that (ii) implies (iii $\mathcal{M}_{\mathcal{M}}$ ).

Summarizing, we have proved that all of conditions (ii), (iii), (iii $\mathcal{M})$, (iv) and (iv $_{\mathcal{M}}$ ) are equivalent with the same constants $c$.

A careful inspection of the proof of Theorem 10 of [8] reveals that the smallest real number $c \geqslant 0$ satisfying (ii) is equal to $\|\widehat{T}\|^{2}$. This and the aforementioned equivalences directly imply (vi). This completes the proof.

The next result improves Theorem 13 of [8] (or rather its counterpart formulated for intertwining operators). It resembles a version of Embry's characterization of subnormality [7] discovered by Lambert in [12]. Recall that a sequence $\left\{a_{n}\right\}_{n=0}^{\infty}$ of 
real numbers is said to be a Hamburger moment sequence if there exists a positive Borel measure $\mu$ on the real line $\mathbb{R}$ such that $a_{n}=\int_{\mathbb{R}} x^{n} \mathrm{~d} \mu(x)$ for all $n \geqslant 0$.

Theorem 3.5. Let $\mathcal{H}_{j}, \mathcal{K}_{j}, S_{j}$ and $N_{j}, j=1,2$, be as in (3.1), and let $T \in$ $\boldsymbol{B}\left(\mathcal{H}_{1}, \mathcal{H}_{2}\right)$. Then the following conditions are equivalent:

(i) $T$ lifts to $\mathcal{I}\left(N_{1}, N_{2}\right)$,

(ii) $T S_{1}=S_{2} T$ and there exists a real number $c \geqslant 0$ such that

$$
\sum_{m, n=0}^{k}\left\langle S_{2}^{m+n} T f_{n}, S_{2}^{m+n} T f_{m}\right\rangle \leqslant c \sum_{m, n=0}^{k}\left\langle S_{1}^{m+n} f_{n}, S_{1}^{m+n} f_{m}\right\rangle
$$

for all finite sequences $\left\{f_{m}\right\}_{m=0}^{k} \subseteq \mathcal{H}_{1}$,

(iii) $T S_{1}=S_{2} T$ and there exists a real number $c \geqslant 0$ such that

$$
\sum_{m, n=0}^{k}\left\|S_{2}^{m+n} T f\right\|^{2} \lambda_{n} \overline{\lambda_{m}} \leqslant c \sum_{m, n=0}^{k}\left\|S_{1}^{m+n} f\right\|^{2} \lambda_{n} \overline{\lambda_{m}}
$$

for every $f \in \mathcal{H}_{1}$ and for all finite sequences $\left\{\lambda_{m}\right\}_{m=0}^{k} \subseteq \mathbb{C}$,

(iv) $T S_{1}=S_{2} T$ and there exists a real number $c \geqslant 0$ such that for every $f \in \mathcal{H}_{1}$, $\left\{c\left\|S_{1}^{n} f\right\|^{2}-\left\|S_{2}^{n} T f\right\|^{2}\right\}_{n=0}^{\infty}$ is a Hamburger moment sequence 1

Moreover, if (i) holds, then

(v) the smallest real number $c \geqslant 0$ satisfying condition (ii) (resp. (iii), (iv)) is equal to $\|\widehat{T}\|^{2}$.

Remark 3.6. Regarding Theorem 3.5 note that conditions (ii), (iii) and (iv) still remain equivalent if we remove the assumption $T S_{1}=S_{2} T$. However, this assumption is indispensable for the validity of (ii) $\Rightarrow(\mathrm{i})$. To see this consider an arbitrary isometry $S \in \boldsymbol{B}(\mathcal{H})$ and an operator $T \in \boldsymbol{B}(\mathcal{H})$ which does not commute with $S$ (cf. Remark 3.4). It is a matter of direct computation that the operators $S_{1}=S_{2}:=S$ and $T$ satisfy (3.5) for all finite sequences $\left\{f_{m}\right\}_{m=0}^{k} \subseteq \mathcal{H}$ (with $c=\|T\|^{2}$ ), though condition (i) is not fulfilled.

Proof of Theorem 3.5. As in the proof of Theorem 3.2 we can assume that the operators $S_{1}$ and $S_{2}$ are contractions.

(i) $\Leftrightarrow($ ii) This is a part of [8, Theorem 13] formulated for intertwining operators.

(ii) $\Rightarrow$ (iii) Applying (3.5) to $f_{n}=\lambda_{n} f$ we get (3.6).

(iii) $\Rightarrow$ (ii) Denote by $\Omega$ the additive $*$-semigroup $(\mathbb{N},+, *)$ with the identity involution $m^{*}=m, m \in \mathbb{N}$. Since (3.6) holds for every $f \in \mathcal{H}_{1}$ and for all finite sequences $\left\{\lambda_{m}\right\}_{m=0}^{k} \subseteq \mathbb{C}$, the operator-valued function $\omega_{c}: \Omega \rightarrow \boldsymbol{B}\left(\mathcal{H}_{1}\right)$ defined by

$$
\omega_{c}(m)=c S_{1}^{* m} S_{1}^{m}-T^{*} S_{2}^{* m} S_{2}^{m} T, \quad m \in \mathbb{N},
$$

is weakly positive definite and uniformly bounded. By Theorem 2.1, $\omega_{c}$ is positive definite, which means that (3.5) holds for all finite sequences $\left\{f_{m}\right\}_{m=0}^{k} \subseteq \mathcal{H}_{1}$.

(iii) $\Leftrightarrow$ (iv) This is a direct consequence of Hamburger's theorem (cf. [2, Theorem 6.2.2] or [17, Theorem 1.2]); the assumption $T S_{1}=S_{2} T$ plays no role.

An inspection of the proof of Theorem 13 of 8 . reveals that the smallest $c \geqslant 0$ satisfying (ii) is equal to $\|\widehat{T}\|^{2}$. The rest of (v) is now easily seen to be true.

\footnotetext{
${ }^{1}$ With a unique representing measure supported in the closed interval $\left[0, \max \left\{\left\|S_{1}\right\|^{2},\left\|S_{2}\right\|^{2}\right\}\right]$ (compare with the proof of Lemma4.1).
} 
The comparison of Theorems 3.2 and 3.5 raises the following

Question. Does the multicyclic version of Theorem 3.5] hold true?

\section{A LOCAL Lifting THEOREM}

For an operator $S \in \boldsymbol{B}(\mathcal{H})$ in a Hilbert space $\mathcal{H}$, we define closed linear spaces:

$$
\begin{aligned}
\mathcal{Q}_{S, f} & =\bigvee\left\{S^{n} f: n \geqslant 0\right\}, \quad f \in \mathcal{H}, \\
\mathcal{Q}^{S, f} & =\bigvee\left\{A_{1} \ldots A_{n} f: A_{1}, \ldots, A_{n} \in\left\{S, S^{*}, I_{\mathcal{H}}\right\}, n \geqslant 1\right\}, \quad f \in \mathcal{H} .
\end{aligned}
$$

It is clear that $\mathcal{Q}_{S, f} \subseteq \mathcal{Q}^{S, f}, \mathcal{Q}_{S, f}$ is the smallest closed linear subspace of $\mathcal{H}$ which is invariant for $S$ and which contains $f$, and $\mathcal{Q}^{S, f}$ is the smallest closed linear subspace of $\mathcal{H}$ which reduces $S$ and which contains $f$. Set $S_{f}=\left.S\right|_{\mathcal{Q}_{S, f}}$ and $S^{f}=\left.S\right|_{\mathcal{Q}^{S, f}}$. It is a matter of routine to show that if $S$ is subnormal and $N \in \boldsymbol{B}(\mathcal{K})$ is a minimal normal extension of $S$, then $\mathcal{Q}_{S, f}=\mathcal{Q}_{N, f}, S_{f}$ is subnormal and $N^{f}$ is a minimal normal extension of $S_{f}$ for every $f \in \mathcal{H}$.

Let $S_{1} \in \boldsymbol{B}\left(\mathcal{H}_{1}\right)$ and $S_{2} \in \boldsymbol{B}\left(\mathcal{H}_{2}\right)$ be operators in Hilbert spaces $\mathcal{H}_{1}$ and $\mathcal{H}_{2}$, respectively. Set $\boldsymbol{S}=\left(S_{1}, S_{2}\right)$. Consider an operator $T \in \boldsymbol{B}\left(\mathcal{H}_{1}, \mathcal{H}_{2}\right)$ such that $T S_{1}=S_{2} T$. For $f \in \mathcal{H}_{1}$, define the operator $T_{\boldsymbol{S}, f} \in \boldsymbol{B}\left(\mathcal{Q}_{S_{1}, f}, \mathcal{Q}_{S_{2}, T f}\right)$ by

$$
T_{\boldsymbol{S}, f} h:=T h, \quad h \in \mathcal{Q}_{S_{1}, f} .
$$

The definition is legitimate because $T S_{1}=S_{2} T$. It is plain to see that

$$
T_{\boldsymbol{S}, f}\left(S_{1}\right)_{f}=\left(S_{2}\right)_{T f} T_{\boldsymbol{S}, f}, \quad f \in \mathcal{H}_{1} .
$$

A (bi)sequence $\left\{c_{m, n}\right\}_{m, n=0}^{\infty} \subseteq \mathbb{C}$ is said to be a complex moment sequence if there exists a positive Borel measure $\mu$ on the complex plane $\mathbb{C}$ (called a representing measure of $\left.\left\{c_{m, n}\right\}_{m, n=0}^{\infty}\right)$ such that $c_{m, n}=\int_{\mathbb{C}} z^{m} \bar{z}^{n} \mathrm{~d} \mu(z)$ for all $m, n \geqslant 0$.

Lemma 4.1. Let $\mathcal{H}_{j}, \mathcal{K}_{j}, S_{j}$ and $N_{j}, j=1,2$, be as in (3.1), and let $T \in$ $\boldsymbol{B}\left(\mathcal{H}_{1}, \mathcal{H}_{2}\right)$ satisfy the equality $T S_{1}=S_{2} T$. Fix a vector $f \in \mathcal{H}_{1}$. Then the following conditions are equivalent:

(i) $T_{\boldsymbol{S}, f}$ lifts to $\mathcal{I}\left(N_{1}^{f}, N_{2}^{T f}\right)$,

(ii) there exists a real number $c \geqslant 0$ such that

$$
\sum_{m, n, p, q=0}^{k}\left\langle S_{2}^{n+p} T f, S_{2}^{m+q} T f\right\rangle \lambda_{m, n} \overline{\lambda_{p, q}} \leqslant c \sum_{m, n, p, q=0}^{k}\left\langle S_{1}^{n+p} f, S_{1}^{m+q} f\right\rangle \lambda_{m, n} \overline{\lambda_{p, q}}
$$

for all finite 2-sequences $\left\{\lambda_{m, n}\right\}_{m, n=0}^{k} \subseteq \mathbb{C}$,

(iii) there exists a real number $c \geqslant 0$ such that the sequence $\left\{c\left\langle S_{1}^{m} f, S_{1}^{n} f\right\rangle-\right.$ $\left.\left\langle S_{2}^{m} T f, S_{2}^{n} T f\right\rangle\right\}_{m, n=0}^{\infty}$ is a complex moment sequence.

Moreover, if (i) holds, then

(iv) $\left\|\widehat{T_{\boldsymbol{S}, f}}\right\|^{2}=\min \{c \geqslant 0: c$ satisfies (ii) $\}=\min \{c \geqslant 0: c$ satisfies (iii) $\}$.

Proof. (i) $\Leftrightarrow$ (ii) Apply Theorem 3.2 to the subnormal operators $\left(S_{1}\right)_{f},\left(S_{2}\right)_{T f}$ intertwined by $T_{\boldsymbol{S}, f}$, to their minimal normal extensions $N_{1}^{f}, N_{2}^{T f}$ and to $\mathcal{M}=\mathbb{C} f$.

(ii) $\Rightarrow$ (iii) Set $a=c\|f\|^{2}+\|T f\|^{2}$ and $b=\max \left\{\left\|S_{1}\right\|,\left\|S_{2}\right\|\right\}$. Since the sequence $c_{m, n}:=c\left\langle S_{1}^{m} f, S_{1}^{n} f\right\rangle-\left\langle S_{2}^{m} T f, S_{2}^{n} T f\right\rangle, m, n \geqslant 0$, is positive definite on the 
*-semigroup $\Omega$ defined in the proof of Theorem 3.2 and it satisfies the estimate

$$
\left|c_{n, n}\right| \leq a b^{2 n}, \quad n \geqslant 0,
$$

we conclude that it is a complex moment sequence having a unique representing measure with support in the closed disc centered at 0 and of radius $b$ (cf. 22, Theorem 4 and Remark 2]). The reverse implication (iii) $\Rightarrow($ ii) is obvious.

We are now ready to prove the main result of the paper.

Theorem 4.2. Let $\mathcal{H}_{j}, \mathcal{K}_{j}, S_{j}$ and $N_{j}, j=1,2$, be as in (3.1). Suppose that $\mathcal{M}$ is a linear subspace of $\mathcal{H}_{1}$ such that $\mathcal{H}_{1}=\bigvee\left\{S_{1}^{n} f: f \in \mathcal{M}, n \in \mathbb{N}\right\}$. Let $T \in \boldsymbol{B}\left(\mathcal{H}_{1}, \mathcal{H}_{2}\right)$. Then the following conditions are equivalent:

(i) $T$ lifts to $\mathcal{I}\left(N_{1}, N_{2}\right)$,

(ii) $T S_{1}=S_{2} T$, the operator $T_{\boldsymbol{S}, f}$ lifts to $\mathcal{I}\left(N_{1}^{f}, N_{2}^{T f}\right)$ for every $f \in \mathcal{M}$, and

$$
\sup _{h \in \mathcal{M}}\left\|\widehat{T_{\boldsymbol{S}, h}}\right\|<\infty
$$

Moreover, if (i) holds, then

$$
\|\widehat{T}\|=\sup _{h \in \mathcal{M}}\left\|\widehat{T_{S, h}}\right\| .
$$

Proof. (i) $\Rightarrow$ (ii) That $T S_{1}$ equals $S_{2} T$ follows from Theorem 3.2 Take a vector $f \in \mathcal{M}$. By the Putnam-Fuglede theorem, we have $\widehat{T} N_{1}^{*}=N_{2}^{*} \widehat{T}$, which together with $\widehat{T} N_{1}=N_{2} \widehat{T}$ and $T \subseteq \widehat{T}$ implies that $\widehat{T}\left(\mathcal{Q}^{N_{1}, f}\right) \subseteq \mathcal{Q}^{N_{2}, T f}$. One can now check that $\left.\widehat{T}\right|_{\mathcal{Q}^{N_{1}, f}} \in \boldsymbol{B}\left(\mathcal{Q}^{N_{1}, f}, \mathcal{Q}^{N_{2}, T f}\right)$ has the following properties:

$$
\left.T_{\boldsymbol{S}, f} \subseteq \widehat{T}\right|_{\mathcal{Q}^{N_{1}, f}} \quad \text { and }\left.\quad \widehat{T}\right|_{\mathcal{Q}^{N_{1}, f}} N_{1}^{f}=\left.N_{2}^{T f} \widehat{T}\right|_{\mathcal{Q}^{N_{1}, f}}
$$

Hence, by the uniqueness of $\widehat{T_{\boldsymbol{S}, f}}$, we must have $\widehat{T_{\boldsymbol{S}, f}}=\left.\widehat{T}\right|_{\mathcal{Q}^{N_{1}, f}}$. As a consequence, the left-hand side of (4.3) is greater than or equal to its right-hand side.

(ii) $\Rightarrow$ (i). Take $f \in \mathcal{M}$. By Lemma 4.1, we have

$$
\sum_{m, n, p, q=0}^{k}\left\langle S_{2}^{n+p} T f, S_{2}^{m+q} T f\right\rangle \lambda_{m, n} \overline{\lambda_{p, q}} \leqslant\left\|\widehat{T_{\boldsymbol{S}, f}}\right\|^{2} \sum_{m, n, p, q=0}^{k}\left\langle S_{1}^{n+p} f, S_{1}^{m+q} f\right\rangle \lambda_{m, n} \overline{\lambda_{p, q}}
$$

for all finite 2-sequences $\left\{\lambda_{m, n}\right\}_{m, n=0}^{k} \subseteq \mathbb{C}$. This and (4.2) combined with the implication $\left(\mathrm{iv}_{\mathcal{M}}\right) \Rightarrow(\mathrm{i})$ of Theorem 3.2 yields (i) and shows that the left-hand side of (4.3) is less than or equal to its right-hand side, which completes the proof.

Proposition 4.3. Let $\mathcal{H}_{j}, \mathcal{K}_{j}, S_{j}$ and $N_{j}, j=1,2$, be as in (3.1). Suppose that $T \in \boldsymbol{B}\left(\mathcal{H}_{1}, \mathcal{H}_{2}\right)$ satisfies the following condition:

(L) $T S_{1}=S_{2} T$ and the operator $T_{\boldsymbol{S}, f}$ lifts to $\mathcal{I}\left(N_{1}^{f}, N_{2}^{T f}\right)$ for every $f \in \mathcal{H}_{1}$. Then the set 2

$$
W_{\boldsymbol{S}, T}:=\left\{f \in \mathcal{H}_{1}: \exists \varepsilon>0 \sup _{h \in \mathcal{H}_{1}:\|h-f\|<\varepsilon}\left\|\widehat{T_{\boldsymbol{S}, h}}\right\|<\infty\right\}
$$

is open and dense in $\mathcal{H}_{1}$.

\footnotetext{
${ }^{2}$ Note that if $f \in W_{\boldsymbol{S}, T}$ and $\varepsilon>0$ is as in (4.4), then the function $\mathcal{H}_{1} \ni h \rightarrow\left\|\widehat{T_{\boldsymbol{S}, h}}\right\|$ is bounded on the open cone $\bigcup_{\lambda \in \mathbb{C} \backslash\{0\}}\left\{h \in \mathcal{H}_{1}:\|h-\lambda f\|<\varepsilon|\lambda|\right\}$ (because $\left\|\widehat{T_{\boldsymbol{S}, \frac{h}{\lambda}}}\right\|=\left\|\widehat{T_{\boldsymbol{S}, h}}\right\|$ ).
} 
Proof. For real $c \geqslant 0$, we denote by $Z_{c}$ the set of all $f \in \mathcal{H}_{1}$ for which the inequality (4.1) holds for all finite 2-sequences $\left\{\lambda_{m, n}\right\}_{m, n=0}^{k} \subseteq \mathbb{C}$. Note that each $Z_{c}$ is closed. By Lemma 4.1, we have

$$
f \in Z_{c} \text { if and only if }\left\|\widehat{T_{\boldsymbol{S}, f}}\right\| \leqslant \sqrt{c} .
$$

Set $U_{h}(\varepsilon)=\left\{f \in \mathcal{H}_{1}:\|f-h\|<\varepsilon\right\}$ for $h \in \mathcal{H}_{1}$ and $\varepsilon>0$. Take $g \in \mathcal{H}_{1}$ and $r>0$. Then $X_{g, n}:=Z_{n} \cap \overline{U_{g}\left(\frac{n}{n+1} r\right)}, n \geqslant 1$, form an increasing sequence of closed sets. It follows from (L) and (4.5) that $U_{g}(r)=\bigcup_{n=1}^{\infty} X_{g, n}$. By the Baire category theorem, there exist $n_{0} \geqslant 1, f_{0} \in X_{g, n_{0}}$ and $\varepsilon>0$ such that $U_{f_{0}}(\varepsilon) \subseteq X_{g, n_{0}} \subseteq Z_{n_{0}}$. This and (4.5) implies that $f_{0} \in W_{\boldsymbol{S}, T} \cap U_{g}(r)$, which completes the proof.

\section{EXAMPLES}

We now illustrate the theme of the paper with three examples. First, we construct an injective operator $T$ intertwining cyclic subnormal operators $S_{1}$ and $S_{2}$, for which the operator $T_{\boldsymbol{S}, f}$ lifts to $\mathcal{I}\left(N_{1}^{f}, N_{2}^{T f}\right)$ if and only if $f=0$.

Example 5.1. Let $\left\{e_{j}\right\}_{j=0}^{\infty}$ be an orthonormal basis of a Hilbert space $\mathcal{H}$. Consider the isometric unilateral shift $S_{1} \in \boldsymbol{B}(\mathcal{H})$ and the weighted shift $S_{2} \in \boldsymbol{B}(\mathcal{H})$ given by $S_{1} e_{j}=e_{j+1}$ and $S_{2} e_{j}=\sqrt{\frac{2 j+1}{2 j+3}} e_{j+1}$ for $j \geqslant 0$. Since

$$
\left\|S_{2}^{j} e_{0}\right\|^{2}=\frac{1}{2 j+1}=\int_{[0,1]} t^{2 j} \mathrm{~d} t, \quad j \geqslant 0,
$$

the Berger-Gellar-Wallen criterion [9, 11] yields the subnormality of $S_{2}$. The operator $T \in \boldsymbol{B}(\mathcal{H})$ defined by $T e_{j}=\frac{1}{\sqrt{2 j+1}} e_{j}, j \geqslant 0$, satisfies the equality $T S_{1}=S_{2} T$. Take $f=\sum_{j=0}^{\infty} \alpha_{j} e_{j} \in \mathcal{H}$. Suppose that $T_{\boldsymbol{S}, f}$ lifts to $\mathcal{I}\left(N_{1}^{f}, N_{2}^{T f}\right)$. By the implication (i) $\Rightarrow$ (ii) of Lemma 4.1, the inequality (3.6) holds for all finite sequences $\left\{\lambda_{m}\right\}_{m=0}^{k} \subseteq \mathbb{C}$. For $k=1, \lambda_{0}=1$ and $\lambda_{1}=-1$ the left-hand side of (3.6) equals

$$
\sum_{j=0}^{\infty}\left|\alpha_{j}\right|^{2}\left(\frac{1}{2 j+1}-\frac{2}{2 j+3}+\frac{1}{2 j+5}\right)=\sum_{j=0}^{\infty} \frac{8\left|\alpha_{j}\right|^{2}}{(2 j+1)(2 j+3)(2 j+5)},
$$

while its right-hand side vanishes. Hence we must have $f=0$.

Next, we examine an example due to Deddens [6]. We show that $T_{\boldsymbol{S}, f}$ lifts to $\mathcal{I}\left(N_{1}^{f}, N_{2}^{T f}\right)$ if and only if $f$ is orthogonal to the cyclic vector $e_{0}$ of $S_{1}$.

Example 5.2. Let $\mathcal{H},\left\{e_{j}\right\}_{j=0}^{\infty}$ and $S_{1}$ be as in Example [5.1, $S_{2}=0$ on $\mathcal{H}$ and $T:=I_{\mathcal{H}}-S_{1} S_{1}^{*}=$ the orthogonal projection of $\mathcal{H}$ onto $\mathbb{C} e_{0}$. Then $T S_{1}=S_{2} T$. Take $f=\sum_{j=0}^{\infty} \alpha_{j} e_{j} \in \mathcal{H}$. If $T f=0$, then condition (ii) of Lemma 4.1 is fulfilled (with $c=0$ ). In turn, if $T f \neq 0$, then for $k=1, \lambda_{0}=1$ and $\lambda_{1}=-1$ the left-hand side of (3.6) is equal to $\|T f\|^{2}>0$, while its right-hand side vanishes.

The third example shows that the implication (ii) $\Rightarrow$ (i) of Theorem 4.2 is no longer true if the hypothesis (4.2) is dropped.

Example 5.3. Let $\varrho_{1}$ be the normalized Lebesgue measure on the unit circle $\mathbb{T}$ and let $\varrho_{2}$ be the one-dimensional Lebesgue measure on the closed interval $\left[0, \frac{1}{2}\right]$. Set $\mu_{n}=\mu=\varrho_{1}+\varrho_{2}$ and $\nu_{n}=\left.\varrho_{2}\right|_{\left[\frac{1}{2^{n+1}}, \frac{1}{2}\right]}$ for all integers $n \geqslant 1$. As usual, $H^{2}$ stands for the Hardy space (both interpretations of $H^{2}$ are exploited; cf. [16]). 
Lemma 5.4. For every $f \in H^{2},\|f\|_{H^{2}} \leqslant\|f\|_{L^{2}(\mu)} \leqslant \sqrt{\frac{5}{3}}\|f\|_{H^{2}}$.

Proof. This can be deduced from the inequality (cf. [10, page 194])

$$
|f(z)| \leqslant \frac{1}{\sqrt{1-|z|^{2}}}\|f\|_{H^{2}} \leqslant \frac{2}{\sqrt{3}}\|f\|_{H^{2}}, \quad z \in \mathbb{C},|z| \leqslant \frac{1}{2}, f \in H^{2} .
$$

Let $\xi(z)=z$ for $z \in \mathbb{C}$. Set $\mathcal{K}_{1}=\bigoplus_{n=1}^{\infty} L^{2}\left(\mu_{n}\right), \mathcal{K}_{2}=\bigoplus_{n=1}^{\infty} L^{2}\left(\nu_{n}\right)$,

$$
\mathcal{H}_{1}=\left\{f=\left(f_{n}\right)_{n=1}^{\infty} \in \mathcal{K}_{1}: f_{n} \in \xi^{n} \cdot H^{2} \text { for all } n \geqslant 1\right\}
$$

and $\mathcal{H}_{2}=\mathcal{K}_{2}$. By Lemma 5.4, $\mathcal{H}_{1}$ is a closed linear subspace of $\mathcal{K}_{1}$. Denote by $M_{\mu_{n}}$ (resp. $M_{\nu_{n}}$ ) the operator of multiplication by $\xi$ on $L^{2}\left(\mu_{n}\right)$ (resp. $L^{2}\left(\nu_{n}\right)$ ). Then $N_{1}:=\bigoplus_{n=1}^{\infty} M_{\mu_{n}}$ and $N_{2}:=\bigoplus_{n=1}^{\infty} M_{\nu_{n}}$ are bounded normal operators on $\mathcal{K}_{1}$ and $\mathcal{K}_{2}$, respectively. It is clear that $N_{1}\left(\mathcal{H}_{1}\right) \subseteq \mathcal{H}_{1}$. Set $S_{1}=\left.N_{1}\right|_{\mathcal{H}_{1}}$ and $S_{2}=N_{2}$. Define the operator $R: \mathcal{K}_{1} \supseteq \mathcal{D}(R) \rightarrow \mathcal{K}_{2}$ as follows:

$$
\begin{gathered}
\mathcal{D}(R)=\left\{f=\left(f_{n}\right)_{n=1}^{\infty} \in \mathcal{K}_{1}: \sum_{n=1}^{\infty} \int\left|\frac{1}{\xi} f_{n}\right|^{2} \mathrm{~d} \nu_{n}<\infty\right\}, \\
R f=\left(\left.\frac{1}{\xi} f_{n}\right|_{\left[\frac{1}{2^{n+1}}, \frac{1}{2}\right]}\right)_{n=1}^{\infty}, \quad f=\left(f_{n}\right)_{n=1}^{\infty} \in \mathcal{D}(R) .
\end{gathered}
$$

It is a routine verification that the operator $R$ is closed, $N_{1}\left(\mathcal{K}_{1}\right) \subseteq \mathcal{D}(R)$ and

$$
R N_{1} f=N_{2} R f, \quad f \in \mathcal{D}(R) .
$$

Set $X=\mathbb{T} \cup\left[0, \frac{1}{2}\right]$. For $\varphi: X \rightarrow \mathbb{C}$ and $f=\left(f_{n}\right)_{n=1}^{\infty} \in \mathcal{K}_{1}$, we write $\varphi f=\left(\varphi f_{n}\right)_{n=1}^{\infty}$.

Lemma 5.5. For every $f \in \mathcal{K}_{1}$,

$$
\mathcal{Q}^{N_{1}, f}=\left\{\varphi f: \varphi \text { complex Borel function on } X, \varphi f \in \mathcal{K}_{1}\right\} .
$$

Proof. Let $\widetilde{\mathcal{Q}}^{N_{1}, f}$ be the right-hand side of (5.3) and $\sigma$ be the Borel measure on $X$ defined by $\mathrm{d} \sigma=\sum_{n=1}^{\infty}\left|f_{n}\right|^{2} \mathrm{~d} \mu$. Since $\sigma(X)=\sum_{n=1}^{\infty}\left\|f_{n}\right\|_{L^{2}\left(\mu_{n}\right)}^{2}=\|f\|_{\mathcal{K}_{1}}^{2}$, the measure $\sigma$ is finite. By the Stone-Weierstrass theorem and [16, Theorems 2.18 and 3.14], the set $\mathbb{C}[z, \bar{z}]$ of all complex polynomials in $z$ and $\bar{z}$ is dense in $L^{2}(\sigma)$. This, the equality $\mathcal{Q}^{N_{1}, f}=\operatorname{closure}\{\varphi f: \varphi \in \mathbb{C}[z, \bar{z}]\}$ and the fact that the map $L^{2}(\sigma) \ni \varphi \mapsto \varphi f \in \widetilde{\mathcal{Q}}^{N_{1}, f}$ is a unitary isomorphism lead to (5.3).

Lemma 5.6. For every $f \in \mathcal{H}_{1}, \mathcal{Q}^{N_{1}, f} \subseteq \mathcal{D}(R)$.

Proof. By (5.3), it suffices to show that if $f=\left(f_{n}\right)_{n=1}^{\infty} \in \mathcal{H}_{1} \backslash\{0\}$ and $\varphi$ is a complex Borel function on $X$ such that $\varphi f \in \mathcal{K}_{1}$, then $\varphi f \in \mathcal{D}(R)$. Let $n_{0} \geqslant 1$ be such that $f_{n_{0}} \neq 0$ and let $\kappa \geqslant 1$ be the order of the zero of $f_{n_{0}}$ at zero. Since $f_{n_{0}} \in H^{2} \backslash\{0\}$, there exists $\varepsilon \in\left(0, \frac{1}{2}\right)$ such that $f_{n_{0}}$ has no zero in $(0, \varepsilon]$. Hence there exists a real number $c>0$ such that

$$
\left|f_{n_{0}}(z)\right| \geqslant c|z|^{\kappa}, \quad z \in[0, \varepsilon] .
$$


It is easily seen that

$$
\begin{gathered}
\int\left|\frac{1}{\xi} \varphi f_{n}\right|^{2} \mathrm{~d} \nu_{n} \leqslant 2^{2(\kappa+1)} \int\left|\varphi f_{n}\right|^{2} \mathrm{~d} \mu_{n}, \quad n=1, \ldots, \kappa \\
\int_{\varepsilon}^{\frac{1}{2}}\left|\frac{1}{\xi} \varphi f_{n}\right|^{2} \mathrm{~d} \nu_{n} \leqslant \frac{1}{\varepsilon^{2}} \int_{\varepsilon}^{\frac{1}{2}}\left|\varphi f_{n}\right|^{2} \mathrm{~d} \nu_{n} \leqslant \frac{1}{\varepsilon^{2}} \int\left|\varphi f_{n}\right|^{2} \mathrm{~d} \mu_{n}, \quad n=1,2, \ldots
\end{gathered}
$$

Since $f_{n} \in \xi^{n} \cdot H^{2}$, there exists $g_{n} \in H^{2}$ such that $f_{n}=\xi^{n} g_{n}$ and $\left\|f_{n}\right\|_{H^{2}}=\left\|g_{n}\right\|_{H^{2}}$. This, (5.1) and (5.4) yield

$$
\left|\frac{1}{z} \varphi(z) f_{n}(z)\right|=|z|^{n-(\kappa+1)}|z|^{\kappa}|\varphi(z)|\left|g_{n}(z)\right| \leqslant 1 \frac{\left|f_{n_{0}}(z)\right|}{c}|\varphi(z)| \frac{2}{\sqrt{3}}\left\|f_{n}\right\|_{H^{2}}
$$

for all $z \in(0, \varepsilon]$ and $n>\kappa$. Thus

$$
\int_{0}^{\varepsilon}\left|\frac{1}{\xi} \varphi f_{n}\right|^{2} \mathrm{~d} \nu_{n} \leqslant c^{\prime} \int_{0}^{\varepsilon}\left|\varphi f_{n_{0}}\right|^{2} \mathrm{~d} \nu_{n}\left\|f_{n}\right\|_{H^{2}}^{2} \leqslant c^{\prime}\|\varphi f\|_{\mathcal{K}_{1}}^{2}\left\|f_{n}\right\|_{L^{2}\left(\mu_{n}\right)}^{2}, \quad n>\kappa,
$$

where $c^{\prime}:=\frac{4}{3 c^{2}}$. Combining (5.5), (5.6) and (5.7), we get $\sum_{n=1}^{\infty} \int\left|\frac{1}{\xi} \varphi f_{n}\right|^{2} \mathrm{~d} \nu_{n}<\infty$, which means that $\varphi f \in \mathcal{D}(R)$ (in fact, we have proved that $\left.R\right|_{\mathcal{Q}^{N_{1}, f}}$ is bounded).

By Lemma 5.6 and the closed graph theorem, the operators $T:=\left.R\right|_{\mathcal{H}_{1}}$ and $\left.R\right|_{\mathcal{Q}^{N_{1}, f}}$ are bounded for all $f \in \mathcal{H}_{1}$. According to (5.2), $T S_{1}=S_{2} T$. Fix $f \in$ $\mathcal{H}_{1}$. By the definition of $R$ and the explicit formulae for $N_{1}^{*}$ and $N_{2}^{*}$, we have $R\left(N_{1}^{* m} N_{1}^{n} f\right)=N_{2}^{* m} N_{2}^{n} R f$ for all $m, n \geqslant 0$. This and the boundedness of $\left.R\right|_{\mathcal{Q}^{N_{1}, f}}$ imply that $R\left(\mathcal{Q}^{N_{1}, f}\right) \subseteq \mathcal{Q}^{N_{2}, R f}$, which together with (5.2) leads to $\left.R\right|_{\mathcal{Q}^{N_{1}, f}} N_{1}^{f}=$ $\left.N_{2}^{R f} R\right|_{\mathcal{Q}^{N_{1}, f}}$. Since $\left.T_{\boldsymbol{S}, f} \subseteq R\right|_{\mathcal{Q}^{N_{1}, f}}$, we conclude that $T_{\boldsymbol{S}, f}$ lifts to $\mathcal{I}\left(N_{1}^{f}, N_{2}^{T f}\right)$ and $\widehat{T_{\boldsymbol{S}, f}}=\left.R\right|_{\mathcal{Q}^{N_{1}, f}}$. We show that $T$ does not lift to $\mathcal{I}\left(N_{1}, N_{2}\right)$. Indeed, if $T$ had a lift $\widehat{T} \in \mathcal{I}\left(N_{1}, N_{2}\right)$, then $\widehat{T}$ would have to agree with $R$ on $\mathcal{E}:=\operatorname{lin} \bigcup_{h \in \mathcal{H}_{1}} \mathcal{Q}^{N_{1}, h}$, because in view of the proof of Theorem $\left.4.2 \widehat{T}\right|_{\mathcal{Q}^{N_{1}, h}}=\widehat{T_{S, h}}=\left.R\right|_{\mathcal{Q}^{N_{1}, h}}$ for all $h \in$ $\mathcal{H}_{1}$. However, the operator $R$ is not bounded on $\mathcal{E}$. Indeed, for $n \geqslant 1,\left(\delta_{n, k} \xi^{n}\right)_{k=1}^{\infty}$ is in $\mathcal{H}_{1}$ and consequently by (5.3) $\left(\delta_{n, k} \xi^{0}\right)_{k=1}^{\infty}=\frac{1}{\xi^{n}} \cdot\left(\delta_{n, k} \xi^{n}\right)_{k=1}^{\infty}$ belongs to $\mathcal{E}$. Since $\left\|R\left(\left(\delta_{n, k} \xi^{0}\right)_{k=1}^{\infty}\right)\right\|_{\mathcal{K}_{2}}^{2}=2^{n+1}-2$ and $\left\|\left(\delta_{n, k} \xi^{0}\right)_{k=1}^{\infty}\right\|_{\mathcal{K}_{1}}^{2}=\frac{3}{2}$ for all $n \geqslant 1,\left.R\right|_{\mathcal{E}}$ is not bounded. Moreover, once again by (5.3),$\left(\delta_{n, k} \varphi\right)_{k=1}^{\infty}=\varphi \frac{1}{\xi^{n}} \cdot\left(\delta_{n, k} \xi^{n}\right)_{k=1}^{\infty} \in \mathcal{E}$ for all $\varphi \in L^{2}\left(\mu_{n}\right)$, which implies that $N_{1}$ is a minimal normal extension of $S_{1}$.

\section{The CASE OF STAR-CYCLIC MINIMAL NORMAL EXTENSIONS}

Let $N \in \boldsymbol{B}(\mathcal{H})$ be a normal operator on a Hilbert space $\mathcal{H}$ and let $E$ be its spectral measure. Recall that $e \in \mathcal{H}$ is a star-cyclic vector for $N$ if $\mathcal{Q}^{N, e}=\mathcal{H}$; denote by $\mathcal{C}_{N}$ the set of all such vectors. If $\mathcal{C}_{N} \neq \varnothing$, then $N$ is called star-cyclic. A vector $e \in \mathcal{H}$ is said to be a separating vector for $N$ if the measures $\langle E(\cdot) e, e\rangle$ and $E$ are mutually absolutely continuous (cf. [5, page 249]). It is clear that $\mathcal{C}_{N}$ is contained in the set of all separating vectors for $N$. If $N$ is star-cyclic, then $e \in \mathcal{H}$ is a star-cyclic vector for $N$ if and only if $e$ is a separating vector for $N$. This is a direct consequence of [4, Theorem IX.3.4] and the following lemma.

Lemma 6.1. If $N$ is the operator of multiplication by the independent variable $z$ on $L^{2}(\mu)$, where $\mu$ is a finite compactly supported Borel measure on $\mathbb{C}$, then

$$
\mathcal{Q}^{N, e}=\left\{\psi e \in L^{2}(\mu): \psi \text { complex Borel function on } \mathbb{C}\right\}=L^{2}\left(\left.\mu\right|_{\operatorname{supp}(e)}\right)
$$


for every $e \in L^{2}(\mu)$, where $\operatorname{supp}(e):=\{w \in \mathbb{C}: e(w) \neq 0\}$, and

(6.2) $\mathcal{C}_{N}=\left\{e \in L^{2}(\mu): e \neq 0\right.$ a.e. $\left.[\mu]\right\}=$ the set of all separating vectors for $N$.

Proof. Adapting the proof of Lemma [5.5. we get the equality appearing on the left-hand side of (6.1). The other equality of (6.1) is easily seen to be true. Since $\langle E(\cdot) e, e\rangle=\int_{(\cdot)}|e|^{2} \mathrm{~d} \mu$, where $E$ is the spectral measure of $N$ (cf. 44, Example IX.2.5]), we verify that $e \in L^{2}(\mu)$ is a separating vector for $N$ if an only if $e \neq 0$ a.e. $[\mu]$. Thus (6.2) results from (6.1). This completes the proof.

Theorem 6.2. Let $\mathcal{H}_{j}, \mathcal{K}_{j}, S_{j}$ and $N_{j}, j=1,2$, be as in (3.1), and let $T \in$ $\boldsymbol{B}\left(\mathcal{H}_{1}, \mathcal{H}_{2}\right)$. Assume that the operators $N_{1}$ and $N_{2}$ are star-cyclic. Suppose $T S_{1}=$ $S_{2} T$ and $T_{\boldsymbol{S}, f}$ lifts to $\mathcal{I}\left(N_{1}^{f}, N_{2}^{T f}\right)$ for all $f \in \mathcal{H}_{1}$. Then $T$ lifts to $\mathcal{I}\left(N_{1}, N_{2}\right)$.

Proof. In view of [4, Theorem IX.3.4], we can assume that $\mathcal{K}_{j}=L^{2}\left(\mu_{j}\right)$, where $\mu_{j}$ is a finite compactly supported Borel measure on $\mathbb{C}$ and $N_{j}$ is the operator of multiplication by the independent variable $z$ on $L^{2}\left(\mu_{j}\right), j=1,2$. Define the set $Y=\left\{z \in \mathbb{C}: h_{1}(z)>0\right.$ and $\left.h_{2}(z)>0\right\}$, where $h_{j}=\mathrm{d} \mu_{j} / \mathrm{d}\left(\mu_{1}+\mu_{2}\right)$ for $j=1,2$.

STEP 1. If $u \in \mathcal{C}_{N_{1}} \cap \mathcal{H}_{1}$, then $T\left(\mathcal{Q}_{S_{1}, u}\right) \subseteq L^{2}\left(\left.\mu_{2}\right|_{Y}\right)$.

Indeed, since $\widehat{T_{\boldsymbol{S}, u}} \in \mathcal{I}\left(N_{1}, N_{2}\right)$, we infer from [1, Theorem 1] that $\widehat{T_{\boldsymbol{S}, u}} f=\varphi_{u} f$ for $f \in L^{2}\left(\mu_{1}\right)$, where $\varphi_{u}$ is a Borel function on $\mathbb{C}$ such that $\varphi_{u}=0$ on $\mathbb{C} \backslash Y$ a.e. $\left[\mu_{2}\right]$. Hence $T\left(\mathcal{Q}_{S_{1}, u}\right) \subseteq \widehat{T_{\boldsymbol{S}, u}}\left(\mathcal{Q}^{N_{1}, u}\right) \subseteq L^{2}\left(\left.\mu_{2}\right|_{Y}\right)$.

STEP 2. $T\left(\mathcal{H}_{1}\right) \subseteq \mathcal{H}_{2} \cap L^{2}\left(\left.\mu_{2}\right|_{Y}\right)$.

By Step 1, $T\left(\mathcal{C}_{N_{1}} \cap \mathcal{H}_{1}\right) \subseteq \mathcal{H}_{2} \cap L^{2}\left(\left.\mu_{2}\right|_{Y}\right)$. Since by [5, Proposition V.17.14] and Lemma 6.1 the set $\mathcal{C}_{N_{1}} \cap \mathcal{H}_{1}$ is dense in $\mathcal{H}_{1}$, we get $T\left(\mathcal{H}_{1}\right) \subseteq \mathcal{H}_{2} \cap L^{2}\left(\left.\mu_{2}\right|_{Y}\right)$.

STEP 3. Reduction of the proof to the case $\mu_{2}=\mu_{1}$.

By Step 2, we can assume that $\mu_{2}=\left.\mu_{2}\right|_{Y}$. Then the measures $\left.\mu_{1}\right|_{Y}$ and $\mu_{2}$ are mutually absolutely continuous and hence the map $U: L^{2}\left(\mu_{2}\right) \ni f \mapsto f \cdot \sqrt{p} \in$ $L^{2}\left(\left.\mu_{1}\right|_{Y}\right)$, where $p:=\mathrm{d} \mu_{2} /\left.\mathrm{d} \mu_{1}\right|_{Y}$, is a unitary isomorphism which intertwines the multiplication operators by $z$ on $L^{2}\left(\mu_{2}\right)$ and $L^{2}\left(\left.\mu_{1}\right|_{Y}\right)$, respectively. This enables us to reduce the general situation to the case $\mu_{2}=\left.\mu_{1}\right|_{Y}$ and then to $\mu_{2}=\mu_{1}$.

From now on, we assume that $\mu_{2}=\mu_{1}=\mu$ and $N_{2}=N_{1}=N$. For $u \in \mathcal{H}_{1}$, we define $\widetilde{T_{\boldsymbol{S}, u}} \in \boldsymbol{B}\left(L^{2}(\mu)\right)$ by $\widetilde{T_{\boldsymbol{S}, u}}=\widetilde{T_{\boldsymbol{S}, u}} \circ P_{u}$, where $P_{u}$ is the orthogonal projection of $L^{2}(\mu)$ onto $\mathcal{Q}^{N, u}$. Then $\widehat{T_{\boldsymbol{S}, u}} \subseteq \widehat{T_{\boldsymbol{S}, u}},\left\|\widehat{T_{\boldsymbol{S}, u}}\right\|=\left\|\widehat{T_{\boldsymbol{S}, u}}\right\|$ and by Lemma 6.1 $\widetilde{T_{\boldsymbol{S}, u}} N=N \widetilde{T_{\boldsymbol{S}, u}}$. Hence there exists a unique $\varphi_{u} \in L^{\infty}(\mu)$ such that

$$
\widetilde{T_{\boldsymbol{S}, u}}(f)=\varphi_{u} \cdot f \text { for all } f \in L^{2}(\mu) \text {, and }\left\|\varphi_{u}\right\|_{L^{\infty}(\mu)}=\left\|\widehat{T_{\boldsymbol{S}, u}}\right\| \text {. }
$$

STEP 4. If $u, v \in \mathcal{C}_{N} \cap \mathcal{H}_{1}$, then $\varphi_{u}=\varphi_{v}$ a.e. $[\mu]$.

Fix real $0<\varepsilon<\mu(\mathbb{C})$. By Lusin's theorem and Lemma 6.1, there exists a compact set $Z_{\varepsilon} \subseteq \mathbb{C}$ such that $\mu\left(\mathbb{C} \backslash Z_{\varepsilon}\right)<\varepsilon$ (hence $\mu\left(Z_{\varepsilon}\right)>0$ ), supp $\left.\mu\right|_{Z_{\varepsilon}}=Z_{\varepsilon}$, the functions $u, v, \varphi_{u}$ and $\varphi_{v}$ are continuous on $Z_{\varepsilon}$, and $u(w) v(w) \neq 0$ for all $w \in Z_{\varepsilon}$. Take $z \in Z_{\varepsilon}$ and set $c_{z}=-\frac{u(z)}{v(z)}$. By (6.3) , there exists a Borel set $X_{0} \subseteq \mathbb{C}$ such that

$$
\left|\varphi_{u+c_{z} v}(w)\right| \leqslant\left\|\widehat{T_{\boldsymbol{S}, u+c_{z} v}}\right\|, \quad w \in X_{0},
$$

and $\mu\left(\mathbb{C} \backslash X_{0}\right)=0$. Applying (6.3) once more we obtain

$$
\varphi_{u+c_{z} v} \cdot\left(u+c_{z} v\right)=T\left(u+c_{z} v\right)=T u+c_{z} T v=\varphi_{u} \cdot u+c_{z} \varphi_{v} \cdot v \quad \text { a.e. }[\mu] .
$$

Diminishing $X_{0}$ if necessary, we can assume that $\mu\left(\mathbb{C} \backslash X_{0}\right)=0$, (6.4) holds and

$$
\varphi_{u+c_{z} v}(w)\left(u(w)+c_{z} v(w)\right)=\varphi_{u}(w) u(w)+c_{z} \varphi_{v}(w) v(w), \quad w \in X_{0} .
$$


Since $z \in Z_{\varepsilon}=\left.\operatorname{supp} \mu\right|_{Z_{\varepsilon}}$, we deduce that $\left\{w \in \mathbb{C}:|w-z|<\frac{1}{n}\right\} \cap Z_{\varepsilon} \cap X_{0} \neq \varnothing$ for every $n \geqslant 1$. Hence there exists $\left\{w_{n}\right\}_{n=1}^{\infty} \subseteq Z_{\varepsilon} \cap X_{0}$ such that $\lim _{n \rightarrow \infty} w_{n}=z$. Substituting $w=w_{n}$ into (6.5), letting $n \rightarrow \infty$ and using (6.4) as well as the continuity of the functions $u, v, \varphi_{u}$ and $\varphi_{v}$ on $Z_{\varepsilon}$ yields

$$
0=\varphi_{u}(z) u(z)+c_{z} \varphi_{v}(z) v(z)=u(z)\left(\varphi_{u}(z)-\varphi_{v}(z)\right) .
$$

Thus $\varphi_{u}(z)=\varphi_{v}(z)$ because $u(z) \neq 0$. Hence $\varphi_{u}=\varphi_{v}$ on $Z_{\varepsilon}$. Since $\varepsilon$ is arbitrary, we conclude that $\varphi_{u}=\varphi_{v}$ a.e. $[\mu]$.

SteP 5. The operator $T$ lifts to $\mathcal{I}(N, N)$.

By [5, Proposition V.17.14], $\overline{\mathcal{C}_{N} \cap \mathcal{H}_{1}}=\mathcal{H}_{1}$. Fix $u \in \mathcal{C}_{N} \cap \mathcal{H}_{1}$. By Step 4, we have

$$
T v=\widehat{T_{\boldsymbol{S}, v}}(v)=\varphi_{v} \cdot v=\varphi_{u} \cdot v=\widehat{T_{\boldsymbol{S}, u}}(v) \quad \text { a.e. }[\mu], \quad v \in \mathcal{C}_{N} \cap \mathcal{H}_{1},
$$

which together with $\overline{\mathcal{C}_{N} \cap \mathcal{H}_{1}}=\mathcal{H}_{1}$ implies that $\widehat{T_{\boldsymbol{S}, u}}$ is the required lift of $T$.

\section{REFERENCES}

[1] M. B. Abrahamse, Some examples on lifting the commutant of a subnormal operator, Ann. Polon. Math., 37 (1980), 289-298. MR0587500 (83e:47013)

[2] C. Berg, J. P. R. Christensen, and P. Ressel, Harmonic Analysis on Semigroups, Springer, Berlin, 1984. MR0747302 (86b:43001)

[3] J. Bram, Subnormal operators, Duke Math. J. 22 (1955), 75-94. MR0068129 (16:835a)

[4] J. B. Conway, A course in functional analysis, Graduate Texts in Mathematics 96, SpringerVerlag, New York, 1990. MR1070713 (91e:46001)

[5] J. B. Conway, The theory of subnormal operators, Mathematical Surveys and Monographs, Providence, Rhode Island, 1991. MR:1112128 (92h:47026)

[6] J. A. Deddens, Intertwining analytic Toeplitz operators, Mich. Math. J. 18 (1971), 243-246. MR0283611 (44:841)

[7] M. Embry, A generalization of the Halmos-Bram criterion for subnormality, Acta Sci. Math. (Szeged) 35 (1973), 61-64. MR0328652 (48:6994)

[8] M. Embry-Wardrop, Quasinormal extensions of subnormal operators Houston J. Math. 7 (1981), 191-204. MR0638946 (83a:47029)

[9] R. Gellar, L. J. Wallen, Subnormal weighted shifts and the Halmos-Bram criterion, Proc. Japan Acad. 46 (1970), 375-378. MR0372672 (51:8879)

[10] P. R. Halmos, A Hilbert Space Problem Book, Van Nostrad, New Jersey, 1967. MR0208368 $(34: 8178)$

[11] P. R. Halmos, Ten problems in Hilbert space, Bull. Amer. Math. Soc. 76 (1970), 887-933. MR0270173 (42:5066)

[12] A. Lambert, Subnormality and weighted shifts, J. London Math. Soc. 14 (1976), 476-480. MR0435915 (55:8866)

[13] P. H. Maserick, Spectral theory of operator-valued transformations, J. Math. Anal. Appl. 41 (1973), 497-507. MR0343084 (49:7828)

[14] W. Mlak, Dilations of Hilbert space operators (General theory), Dissertationes Math. 153 (1978), 1-65. MR0496046 (81j:47008)

[15] C. R. Putnam, On normal operators in Hilbert space, Amer. J. Math. 73 (1951), 357-362. MR0040585 (12:717f)

[16] W. Rudin, Real and Complex Analysis, McGraw-Hill, New York, 1974. MR0344043 (49:8783)

[17] J. A. Shohat and J. D. Tamarkin, The problem of moments, Math. Surveys 1, Amer. Math. Soc., Providence, Rhode Island, 1943. MR0008438 (5:5c)

[18] J. Stochel, The Bochner type theorem for $*$-definite kernels on abelian $*$-semigroups without neutral element, Operator Theory: Adv. Appl. 11, Birkhäuser, Basel 1983, 345-362. MR0789648 (86m:47007)

[19] J. Stochel, Characterizations of subnormal operators, Studia Math. 97 (1991), 227-238. MR1100688 (92m:47047)

[20] J. Stochel, Decomposition and disintegration of positive definite kernels on convex *semigroups, Ann. Polon. Math. 56 (1992), 243-294. MR.1160416 (93g:43003) 
[21] J. Stochel, Lifting strong commutants of unbounded subnormal operators, Integral Equations and Operator Theory 43 (2002), 189-214. MR1894622 (2003g:47035)

[22] F. H. Szafraniec, Boundedness of the shift operator related to positive definite forms: an application to moment problems, Ark. Mat. 19 (1981), 251-259. MR0650498 (84b:44015)

[23] T. Yoshino, A note on a result of J. Bram, Duke Math. J. 43 (1976), 875. MR0415379 $(54: 3467)$

Faculty of Applied Mathematics, AGH Science and Technology University, Al. Mickiewicza 30, 30-059 Kraków, Poland

E-mail address: majdak@wms.mat.agh.edu.pl

Department of Applied Analysis, Eötvös University, H-1117 Budapest, Pázmány Péter SÉTÁNY 1/C, HUNGARY

E-mail address: sebesty@cs.elte.hu

Instytut Matematyki, Uniwersytet Jagielloński, Reymonta 4, 30-059 Kraków, Poland

E-mail address: stochel@im.uj.edu.pl

Department of Mathematics, 460 McBryde Hall, Virginia Tech, Blacksburg, Virginia 24061-0123

E-mail address: thomson@math.vt.edu 